\title{
Domain-specific learning of grammatical structure in musical and phonological sequences
}

\author{
Benjamin Martin BLy \\ New Jersey Medical School, Newark, New Jersey \\ RICARDo E. CARrión \\ Robert Wood Johnson Medical School, New Brunswick, New Jersey \\ AND \\ BJÖRN RASCH \\ University of Lübeck, Lübeck, Germany
}

\begin{abstract}
Artificial grammar learning depends on acquisition of abstract structural representations rather than domainspecific representational constraints, or so many studies tell us. Using an artificial grammar task, we compared learning performance in two stimulus domains in which respondents have differing tacit prior knowledge. We found that despite grammatically identical sequence structures, learning was better for harmonically related chord sequences than for letter name sequences or harmonically unrelated chord sequences. We also found transfer effects within the musical and letter name tasks, but not across the domains. We conclude that knowledge acquired in implicit learning depends not only on abstract features of structured stimuli, but that the learning of regularities is in some respects domain-specific and strongly linked to particular features of the stimulus domain.
\end{abstract}

The recognition of patterns in space and time organizes the environment and its dynamics, making them understandable and informative. An influential theoretical position proposes that the learning of nonsalient covariances in the environmental input is performed by a distinct learning mechanism called implicit learning (Berry \& Dienes, 1991; Cleeremans, Destrebecqz, \& Boyer, 1998; A. S. Reber, 1993). The implicit learning process is regarded as independent of intention, and the knowledge acquired from implicit learning is taken to be a tacit, abstract representation not amenable to verbal description (A. S. Reber, 1989).

\section{Implicit Learning Tasks}

Implicit learning paradigms generally expose participants to complex rule-governed stimuli without instructions about the existence of the underlying rules. Then participants are tested for evidence of incidentally acquired knowledge about the structure of the material that improves their performance in the same task or a different one.

In an artificial grammar task, the underlying rules correspond to a finite state machine that defines the set of units and their positions in a sequence. In some of the first artificial grammar experiments, A. S. Reber (1967) observed that participants were able to distinguish between novel letter strings generated from the same finite state machine and "ungrammatical" letter sequences. These findings have been replicated and extended by a number of researchers since A. S. Reber's initial studies (for reviews, see A. S. Reber, 1989, 1993; Reed \& Johnson, 1994; Vokey \& Brooks, 1992).

Alternatives to A. S. Reber's abstractionist account include chunking or fragment-based approaches (Gomez, 1997; Knowlton \& Squire, 1996; Perruchet \& Pacteau, 1990; Servan-Schreiber \& Anderson, 1990) and associative learning approaches (Altmann, Dienes, \& Goode, 1995; Cleeremans \& McClelland, 1991; Dienes, Altmann, \& Gao, 1999).

\section{Novel Versus Familiar Domains}

Implicit learning models generally have arisen from empirical investigations employing abstract elements (e.g., letters, symbols, and colors) in arbitrary juxtapositions determined by artificial rules to study pattern learning in contexts semantically impoverished by design. Outside the lab, however, the meaningful learning of regularities generally occurs in richly represented situations. In the present research, we compared participants' performance in a domain in which they already have tacit, implicitly acquired knowledge of regularities in the stimulus environment with their performance in a novel domain.

\section{Prior Knowledge of Musical Harmony}

We tested learning in the domain of musical harmony, which, like language, is a highly structured system that may be learned in an incidental manner (Tillmann, Bharucha, \& Bigand, 2000), and about which people without 
formal training are well able to acquire knowledge without being able to formulate the explicit rules governing the forms (Aldwell \& Schachter, 2003; Kostka \& Payne, 2000). As in language, the grammar of tonal music creates expectations about impending events in the listener (Besson \& Schön, 2003; Bharucha \& Stoeckig, 1986).

One form of expectation created by music concerns harmony. For example, sequences of chords in Western tonal music normally begin with a specific and consonant chord, proceed to more dissonant chords, and are resolved by a consonant chord. This structure can be found in numerous classical musical pieces as well as in almost every song in pop, rock, and jazz, but, as in language, it is difficult for nonmusicians to describe the harmonic structure of such and such a cadence, or to verbalize the rules.

In addition to a large literature of behavioral research showing that individuals have implicit knowledge of harmonic structure (Bharucha \& Stoeckig, 1986, 1987; Tekman \& Bharucha, 1992, 1998; Tillmann et al., 2000), recent neuroscientific research has shown the same (Besson \& Faïta, 1995; Crummer, Walton, Wayman, Hantz, \& Frisina, 1994; Koelsch, Gunter, Friederici, \& Schröger, 2000; Koelsch, Gunter, Schröger, Tervaniemi, \& Friederici, 2001; Koelsch, Jentschke, Sammler, \& Mietchen, 2007; Koelsch, Maess, Grossmann, \& Friederici, 2003; Steinbeis, Koelsch, \& Sloboda, 2006; Tervaniemi, Ilvonen, Karma, Alho, \& Näätänen, 1997). In our own work, we have found an ERP response to unexpected musical events that takes the form of a specific, predictable waveform complex (Carrión \& Bly, 2008).

We sought to compare learning in two domains: one, a familiar domain in which participants could be expected to have abstracted knowledge of regularities, and the second domain, the arbitrary and decontextualized domain of letter name sequences.

\section{EXPERIMENT 1A}

The aim of Experiment 1A was to determine whether background knowledge of musical harmony could facilitate learning in an artificial grammar task, in comparison with a contextually arbitrary domain in which no background knowledge was available to facilitate learning.

\section{Method}

\section{Participants}

The participants were 202 undergraduate students at Rutgers Newark University who participated in the experiment for course credit or as a course requirement. Participants were nonmusicians; that is, no participant had any formal musical training (besides normal grammar school musical education) or reported knowing how to play a musical instrument of any kind. They were randomly assigned to one of four experimental conditions: harmonically related chord sequences ( $n=50 ; 15$ males, 35 females), harmonically unrelated chord sequences ( $n=50 ; 23$ males, 27 females), letter name sequences that corresponded exactly to the harmonically related chord sequences ( $n=51 ; 17$ males, 34 females), or letter name sequences that corresponded exactly to the harmonically unrelated chord sequences ( $n=51 ; 20$ males, 31 females).

\section{Stimuli}

We used two domains (musical chords and spoken letter names). Sequences varied from four to nine chords or letter names in length. Chords were played by a MIDI keyboard. Letter names were spoken by a male speaker blind to the experimental hypothesis and carefully controlled for speech intonation. Chords and letters were played at a speed of $600 \mathrm{msec}$ per element, resulting in a sequence length of 3 to $6 \mathrm{sec}$.

Harmonically related grammatical sequences. Harmonically related grammatical sequences were composed of five chords harmonically related to the key of C Major: C Major (C), D Minor (Dm), F Major (F), G7, and A Minor (Am). Grammatical sequences always started and ended on the tonic chord C. Three different chords were possible at the second position: Dm, F, or Am. A G7 chord (unstable dominant seventh) was always preceded by Dm, F, or Am, and followed by a stable $\mathrm{C}$ Major chord. This positional regularity was likely to be consistent with our participants' tacit knowledge of harmonic structure, since it obeys conventions of Western musical harmony. The finite-state machine that produced grammatical sequences is shown in Figure 1.

The grammatical letter name strings that corresponded to the related chord sequences were constructed by substituting one letter name for each of the five chords (C:M, Am:S, Dm:W, F:X, and G7:R). We thus obtained two sets of grammatical sequences with exactly the same underlying rules, statistical properties, and chunk strength.

We were mindful that any performance advantage conferred on the chord grammars over the letter grammars might come from gen-

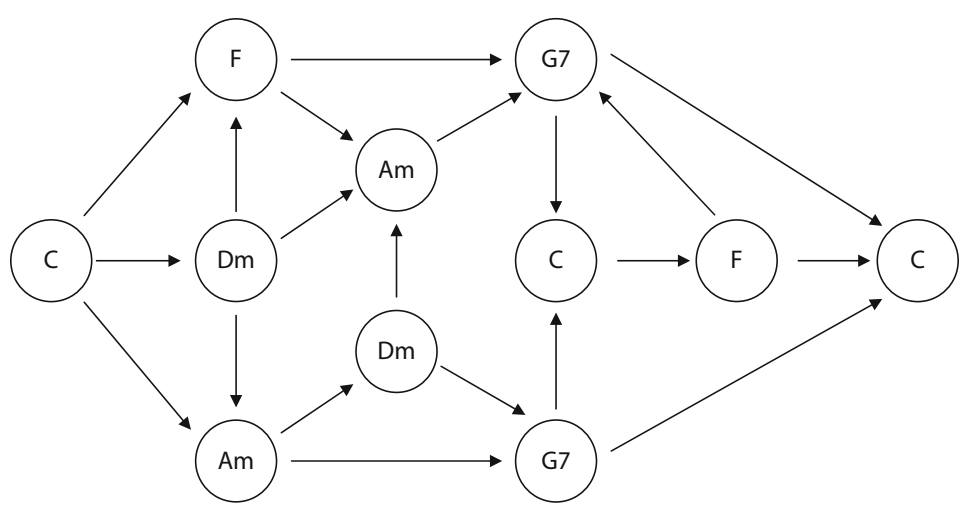

Figure 1. Machine A, the finite state machine used to construct all grammatical sequences in Experiments 1 ( $A$ and $B$ ) and 2. It is shown with the vocabulary used to produce harmonically related chord sequences. 


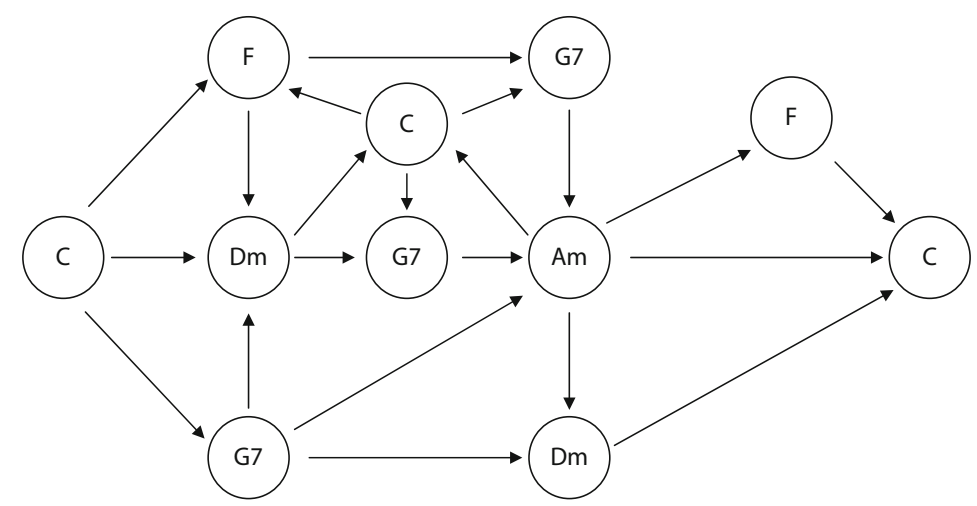

Figure 2. Machine B, the finite state machine used to construct all ungrammatical sequences in Experiments 1 ( $A$ and $B$ ) and 2. It is shown with the vocabulary used to produce harmonically related chord sequences.

eral properties of music, or from musical processing mechanisms in the brain. Although both chords and letters were to be presented auditorily at the rate of $600 \mathrm{msec}$, the possibility existed that processing chord sequences would itself be perceptually or computationally easier than processing letter sequences would be. To show that the performance advantage conferred on the chord grammars came from specific tacit knowledge of the musical domain rather than from general properties of music, or from musical processing mechanisms in the brain, we included two additional conditions.

Harmonically unrelated grammatical sequences. In a second chord condition, we constructed the chord sequences in such a way that previously acquired tacit knowledge would not aid performance. Western music shares certain general characteristics that constrain what Westerners hear as harmonically related. Therefore, we constructed sequences that violated Western musical norms: harmonically unrelated chord sequences, using the same finite state machine shown in Figure 1, but merely substituting $C^{\sharp}$ for $C$ and $A \sharp$ for $A m$. These sequences were not as well formed as the harmonically related chord progressions were, and they sounded disjointed and arbitrary. Each one, however, was grammatical in the sense that it was formed from Machine A and was equivalent to a harmonically related grammatical sequence also formed from that machine.

We also created a set of letter name sequences in the same way as we had for the harmonically related condition: We replaced the unrelated chord names with letter names. Letter name sequences corresponding to the unrelated chord sequences were composed of the spoken names of the letters $\mathrm{L}, \mathrm{W}, \mathrm{X}, \mathrm{Q}$, and $\mathrm{S}$, using $\mathrm{L}$ for $\mathrm{C}^{\sharp}$ and $\mathrm{Q}$ for $\mathrm{A}^{\sharp}$.

Harmonically related ungrammatical sequences. We paid close attention to the creation of the related ungrammatical sequences. Various researchers have asserted that implicit learning results can be explained by participants' detection of violations. By this argument, the bigrams and trigrams are not more salient in the gram- matical sequences, but the violations of bigrams and trigrams are more salient in the ungrammatical sequences. In designing the study, we attempted to circumvent this problem by using a separate finite state machine to generate ungrammatical strings, rather than creating ungrammatical strings merely by violating the rules of the finite state machine that generated grammatical strings. In this way, the ungrammatical strings were based on a coherent, rule-based abstraction, just as the grammatical strings were, but they were "ungrammatical" in the special sense that they could not be generated by Machine A.

Machine B (shown in Figure 2) shared many of the constraints of Machine A and used exactly the same five chords as did Machine A.

For ungrammatical sequences, the first and last chord was always $\mathrm{C}$ Major, as in the grammatical sequences. However, none of the ungrammatical sequences included the bigram G7-C; instead, G7 was always followed by another chord in the ungrammatical sequences (usually Am, sometimes Dm). Musically, this can still be understood as a resolution. Therefore, the ungrammatical sequences did not sound harmonically ill formed (as the harmonically unrelated chord progressions did), even though the resolution to Am might be less expected by listeners of modern Western music than the more frequently used resolution to C Major.

We used the same substitution rules to construct ungrammatical letter name sequences as we had done for the grammatical sequences (C:M, Am:S, Dm:W, F:X, and G7:R). We thus had the same five chords used in grammatical and ungrammatical chord sequences, and the same five letters used in the grammatical and ungrammatical letter sequences.

Harmonically unrelated ungrammatical sequences. For a harmonically unrelated chord condition, we constructed the ungrammatical chord sequences using the same finite state machine (Figure 2), again substituting $C \sharp$ for $C$ and $A \sharp$ for Am. Letter sequences corresponding to the unrelated chord sequences were composed of the letters $\mathrm{L}, \mathrm{W}, \mathrm{X}, \mathrm{Q}$, and $\mathrm{S}$, using $\mathrm{L}$ for $\mathrm{C}^{\sharp}$ and $\mathrm{Q}$ for $\mathrm{A}^{\sharp}$.

Table 1

Sequence Generation Summary

\begin{tabular}{|c|c|c|c|c|}
\hline & \multicolumn{4}{|c|}{ Machine } \\
\hline & \multicolumn{2}{|c|}{$\begin{array}{c}\text { A } \\
\text { Grammatical }\end{array}$} & \multicolumn{2}{|c|}{$\begin{array}{c}\text { B } \\
\text { Ungrammatical }\end{array}$} \\
\hline & Domain & Items & Domain & Items \\
\hline $\begin{array}{l}\text { Harmonically } \\
\text { related }\end{array}$ & $\begin{array}{l}\text { Chords } \\
\text { Letters }\end{array}$ & $\begin{array}{l}\text { C, F, G7, Am, Dm } \\
\text { M. S, W, R. X }\end{array}$ & $\begin{array}{l}\text { Chords } \\
\text { Letters }\end{array}$ & $\begin{array}{l}\text { C, F, G7, Am, Dm } \\
\text { M, S, W, R, X }\end{array}$ \\
\hline $\begin{array}{c}\text { Harmonically } \\
\text { unrelated }\end{array}$ & $\begin{array}{l}\text { Chords } \\
\text { Letters }\end{array}$ & $\begin{array}{l}\mathrm{C} \#, \mathrm{~F}, \mathrm{G} 7, \mathrm{~A}^{\sharp}, \mathrm{Dm} \\
\mathrm{L}, \mathrm{S}, \mathrm{W}, \mathrm{Q}, \mathrm{X}\end{array}$ & $\begin{array}{l}\text { Chords } \\
\text { Letters }\end{array}$ & $\begin{array}{l}C^{\sharp}, F, G 7, A^{\sharp}, D m \\
L, S, W, Q, X\end{array}$ \\
\hline
\end{tabular}


Table 1 summarizes the items (letters and chords) and sequences (harmonically related chords, harmonically unrelated chords, harmonically related letter names, harmonically unrelated letter names) used in all the experiments.

From the total set of possible grammatical sequences (Machine A), we selected 18 sequences as training sequences across all four conditions. The training sequences were also presented in the test phase, with an additional 18 grammatical sequences that the participants had not heard during training, for a total of 36 grammatical sequences during the test phase. From the sequences generated by Machine B, we selected a total number of 36 ungrammatical sequences matched to the grammatical sequences in the number of chords, the total frequency of occurrence of each chord or letter, and the frequency of occurrence of each chord/letter at any particular position, as closely as possible.

In all conditions, then, the single finite state Machine A (Figure 1) generated all the grammatical sequences, and a single finite state Machine B (Figure 2) produced all the ungrammatical sequences. The selected grammatical sequences were always the same across the four conditions, so the statistical properties of frequency and bigram and trigram positions and violations were exactly the same for all conditions. The selected ungrammatical sequences were also the same across all conditions and the potential for detecting violations was therefore equivalent across all conditions.

\section{Design}

The study was planned as a 2 (harmonically related vs. harmonically unrelated) $\times 2$ (letters vs. chords) between-subjects design, so that the two letter name controls would perfectly match the two chord conditions. (The distinction "harmonic relatedness" or "relatedness," although relevant only to the chord sequences, was retained in order to make the relevant comparisons clear.) The final design, therefore, had four between-subjects conditions.

\section{Procedure}

Participants were informed in a consent form about the general goals of the research, confidentiality, and their general rights as participants in a human subject experiment. Letter name and chord sequences were presented via headphones and controlled via the experiment software E-Prime. Participants were instructed that they would hear sequences of letters or chords and that they should listen carefully, because they would be asked questions about the sequences. The volume was adjusted to a comfortable level. After hearing a sequence in the training phase, participants pressed the space bar to hear the next sequence. During training, the 18 grammatical sequences were repeated four times in a random order; in total, the training phase consisted of 72 sequences.

After a 1-min break, participants were informed that the sequences that they had heard before were constructed by a set of rules. They were told that they would hear sequences of letters and chords again and that they should respond "yes" if they thought that a particular sequence respected the same set of rules as the sequences they had heard before. They should press "no" if they thought that the particular sequence was constructed by a different set of rules. They were informed that they should trust their "gut feelings" for answering. After each trial, participants provided an estimate of their detection performance in the form of confidence ratings, varying from random guessing to complete confidence using the numbers 1 to 4 , where 1 meant $I$ am guessing and 4 meant $I$ am sure. The test phase encompassed 36 grammatical and 36 ungrammatical sequences, for a total of 72 sequences presented in random order.

After training and testing, participants filled out a questionnaire that contained general demographic questions and specific items about musical preferences, abilities, and musical training. They also provided confidence estimates of their overall classification performance on a 4-point scale $(1,50 \% ; 2,50 \%-70 \% ; 3,70 \%-90 \%$; 4 , more than $90 \%$ ). They indicated the most important difference between the grammatical and ungrammatical sequences, the method that they used to perform the tasks, and particular elements most salient to them.

\section{Results}

The performance of the participants, gauged by the percent of stimuli correctly classified, is shown in Fig-

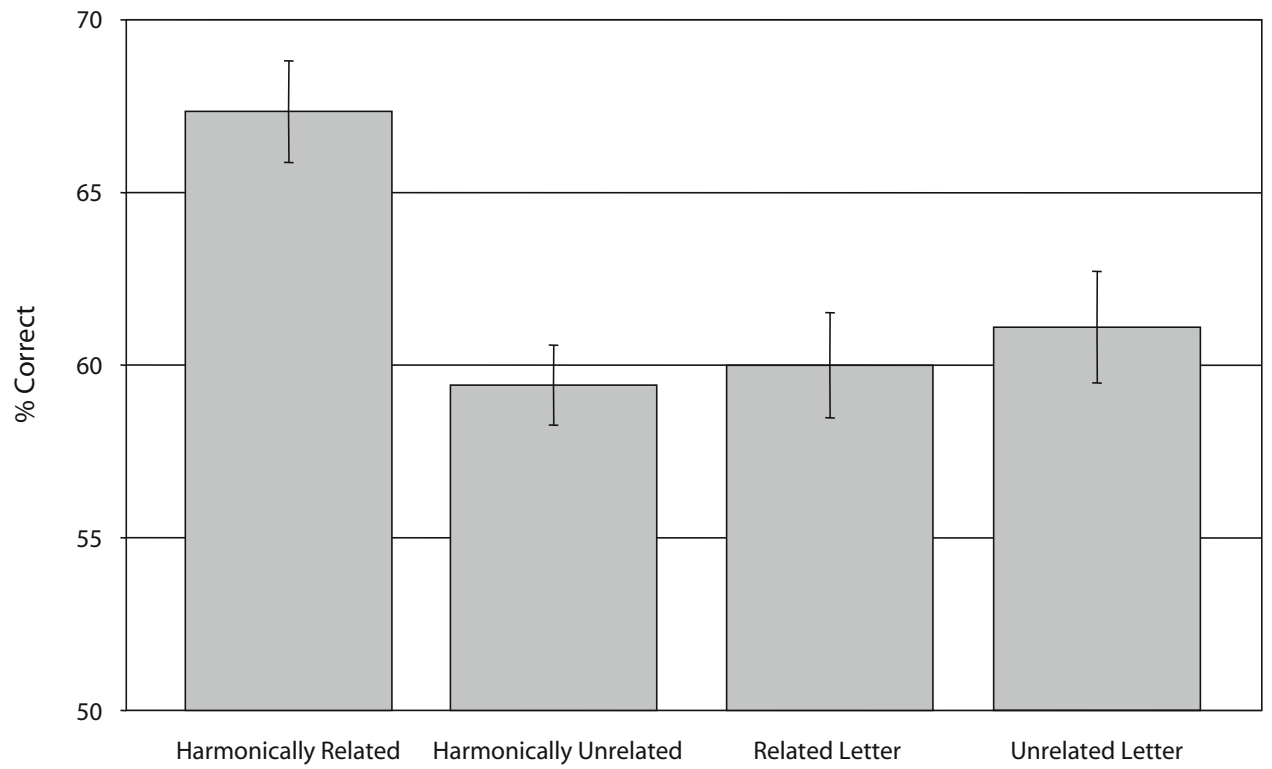

Condition

Figure 3. Accuracy of sequence classification in Experiment $1 \mathrm{~A}$. 
Table 2

Experiment 1A: Hits and Correct Rejections

\begin{tabular}{|c|c|c|c|c|}
\hline \multirow[b]{2}{*}{ Condition } & \multicolumn{2}{|c|}{ Hits } & \multicolumn{2}{|c|}{$\begin{array}{c}\text { Correct } \\
\text { Rejections }\end{array}$} \\
\hline & M & $S D$ & M & $S D$ \\
\hline Harmonically related chords & 74.5 & .14 & 60.1 & .19 \\
\hline Harmonically unrelated chords & 73.1 & .15 & 45.5 & .21 \\
\hline Related letters & 66.8 & .13 & 52.9 & .21 \\
\hline Unrelated letters & 69.0 & .18 & 53.1 & .21 \\
\hline
\end{tabular}

ure 3. Error bars represent one standard error. A one-way ANOVA performed on the mean classification performance of the four conditions revealed a significant effect of experimental condition $\left[F(3,198)=6.27, M S_{\mathrm{e}}=\right.$ $.011, p<.001]$. Planned comparisons revealed that performance in the related chord condition was significantly higher than was performance in the unrelated chord condition $[t(198)=3.84, p=.001, d=.08]$, related letter sequences $[t(198)=3.58, p=.001, d=.07]$, and unrelated letter sequences $[t(198)=3.04, p=.01, d=.06]$. The contrasts also revealed that the performance levels seen in the unrelated chord condition, related letter sequences, and unrelated letter sequences were statistically equivalent. We did not find any sex differences.

In all experimental conditions, performance was statistically above chance (50\%). Participants performed in the related chord condition at a $67.3 \%(S D=.10)$ discrimination level $[t(49)=11.79, p<.001, d=.17]$. Participants in the unrelated chord condition performed at a $59.4 \%$ $(S D=.08)$ discrimination level $[t(49)=8.15, p<.001$, $d=.09]$. Participants in the related letter sequences condition performed at a $60.0 \%(S D=.11)$ discrimination level $[t(50)=6.59, p<.001, d=.10]$. In the unrelated letter sequences condition, participants performed at a $61.1 \%(S D=.12)$ discrimination level $[t(50)=6.88, p<$ $.001, d=.11]$.

We examined our results closely to determine whether the detection of violations was driving our effects (see Table 2). We calculated the percentage of correct classification of a grammatical sequence (hits) versus the percentage of correctly identifying a sequence as ungrammatical (correct rejections). We calculated a repeated measures ANOVA with separate probabilities for hits versus correct rejections. This new factor was highly significant $\left[F(1,198)=71.29, M S_{\mathrm{e}}=.044, p<.001\right]$, but the interaction with experimental condition was not $\left[F(3,198)=1.82, M S_{\mathrm{e}}=.044, p=.15\right]$. The percentage of hits $(70.7 \%)$ was higher than the percentage of correct rejections $(53.0 \%)$, but this difference was equivalent across all conditions. It was not the case, then, that the observed higher performance in the sequences with related chords was caused only by a higher rate of rejection of the ungrammatical sequences.

\section{Awareness Data}

For each experiment, participants' awareness of the acquired knowledge was assessed by looking at the zero correlation criterion (see Chan, 1992; Dienes \& Altmann,
1997; Dienes, Altmann, Kwan, \& Goode, 1995), the guessing criterion (see Cheesman \& Merikle, 1984; Dienes et al., 1995), estimates of overall classification performance, and participants' verbal reports of performance. Participants' subjective awareness was only analyzed for conditions in which learning was observed.

Table 3 displays the mean confidence ratings for each of the conditions. A one-way ANOVA showed no significant differences across the four groups $[F(3,198)=0.34$, $\left.M S_{\mathrm{e}}=.346, p=.80\right]$. Neither were estimates of detection performance given by the participants significantly correlated with performance for any condition: related chords $[r(48)=.25, p=.10]$, unrelated chords $[r(48)=-.02$, $p=.90]$, related letter sequences $[r(49)=.27, p=.06]$, and unrelated letter sequences $[r(49)=.05, p=.75]$.

Table 4 displays the mean difference in confidence between correct decisions and incorrect decisions. A oneway ANOVA performed on the difference scores revealed marginal significant differences $\left[F(3,198)=2.58, M S_{\mathrm{e}}=\right.$ $.346, p=.06]$. However, each condition showed significant differences from zero: related chords $[t(49)=7.86$, $p<.001, d=.27]$, unrelated chords [t(49) $=5.02, p<$ $.001, d=.17]$, related letter sequences $[t(50)=3.22, p<$ $.001, d=.13]$, and unrelated letter sequences $[t(50)=$

Table 3

Mean Confidence Ratings

\begin{tabular}{|c|c|c|}
\hline Condition & $M$ & $S D$ \\
\hline \multicolumn{3}{|l|}{ Experiment $1 \mathrm{~A}$} \\
\hline Harmonically related chords & 2.89 & .53 \\
\hline Harmonically unrelated chords & 2.82 & .58 \\
\hline Related letters & 2.79 & .59 \\
\hline Unrelated letters & 2.79 & .64 \\
\hline \multicolumn{3}{|l|}{ Experiment 2} \\
\hline $\begin{array}{l}\text { Harmonically related chords to } \\
\text { harmonically unrelated chords }\end{array}$ & 2.42 & .58 \\
\hline Related letters to unrelated letters & 2.61 & .65 \\
\hline Unrelated letters to related letters & 2.62 & .61 \\
\hline
\end{tabular}

Note-Data are presented as mean estimates of detection performance in the form of confidence ratings. Mean confidence ratings were only calculated in cases where there was evidence of learning.

Table 4

Zero-Correlation Criterion: Mean Difference in Confidence

\begin{tabular}{lll}
\hline \multicolumn{1}{c}{ Condition } & $M$ & $S D$ \\
\hline \multicolumn{1}{c}{$\begin{array}{l}\text { Experiment 1A } \\
\text { Harmonically related chords }\end{array}$} & .27 & .24 \\
Harmonically unrelated chords & .17 & .24 \\
Related letters & .13 & .28 \\
Unrelated letters $\quad$ Experiment 2 & .15 & .32 \\
Harmonically related chords to & & \\
harmonically unrelated chords & .03 & .21 \\
Related letters to unrelated letters & .08 & .27 \\
Unrelated letters to related letters & .08 & .22
\end{tabular}

Note-Data are presented as the mean difference in confidence ratings between when subjects made a correct decision and when they made an incorrect decision. The zero-correlation criterion was only calculated in cases where there was evidence of learning. 
Table 5

Guessing Criterion

\begin{tabular}{lcc}
\hline \multicolumn{1}{c}{ Condition } & $M$ & $S D$ \\
\hline \multicolumn{1}{c}{$\begin{array}{c}\text { Experiment 1A } \\
\text { Harmonically related chords }\end{array}$} & 55.3 & .27 \\
Harmonically unrelated chords & 51.4 & .22 \\
Related letters & 58.3 & .29 \\
Unrelated letters & 56.9 & .26 \\
& & \\
Harmonically related chords to & & \\
harmonically unrelated chords & 54.1 & .17 \\
Related letters to unrelated letters & 53.6 & .21 \\
Unrelated letters to related letters & 49.6 & .22 \\
\hline
\end{tabular}

Note-Data are presented as mean classification responses for trials where participants were guessing. The guessing criterion was only calculated in cases where there was evidence of learning.

3.47, $p<.001, d=.15]$. Therefore, participants' greater certainty in their decisions when they were right than when they were wrong was not related to a particular experimental condition.

Table 5 shows the mean classification performance when participants were guessing (i.e., gave a confidence rating of 1). A one-way ANOVA found no significant differences $\left[F(3,148)=2.58, M S_{\mathrm{e}}=.07, p=.68\right]$. Classification performance when participants were guessing was not significantly different from chance for any condition: related chords $[t(32)=1.14, p=.26]$, unrelated chords $[t(36)=.39, p=.70]$, related letter sequences $[t(41)=$ $1.88, p=.07]$, and unrelated letter sequences $[t(39)=$ $1.67, p=.10]$. Participants knew when they were applying knowledge and when they were just guessing; this implies that they did not acquire implicit knowledge.

A one-way ANOVA comparing participants' estimates of percent correct classification revealed no significant differences $\left[F(3,194)=0.77, M S_{\mathrm{e}}=.52, p=.51\right]$, nor were estimates significantly correlated with performance for any condition: related chords $[r(48)=.26, p=.08]$, unrelated chords $[r(48)=-.01, p=.96]$, related letter sequences $[r(49)=.14, p=.33]$, and unrelated letter sequences $[r(49)=.21, p=.14]$. In spite of the higher performance in the related chords condition, participants were not better in assessing their overall performance in this task.

Participants' self-reports suggested that they were mostly unaware of the differences between the grammatical and ungrammatical sequence sets. Three very high performers in the letters condition reported noticing the different trigrams necessary for solving the task, but in spite of the fact that knowledge about two starting trigrams and three ending trigrams was in principle enough to achieve $100 \%$ performance, no one achieved anything close to this level of performance. In the music conditions, a few participants reported that they listened especially closely to the starting and ending patterns of the sequences, and some tried to describe the sequence as a pattern of high and low pitches, but in general they did not provide descriptions of any regularities as clearly as did participants in the letter conditions. None of them was able to describe the necessary chord patterns as trigrams in harmonic terms and no differences were found between descriptions in the harmonically related versus harmonically unrelated conditions.

\section{Discussion}

Performance levels for the harmonically unrelated chord sequences and both the letter name conditions were consistent with many artificial grammar learning results in the literature: never close to $100 \%$, but above chance.

Importantly, our results show that participants performed better when the sequences consisted of harmonically related chords than when they were composed of identically structured harmonically unrelated chords or identically structured letter strings. Since both kinds of chord sequences and letter strings were formed from the same finite state machine, the vocabulary items and statistical properties of frequency or chunk strength were the same in all four conditions.

Our belief is that these results show two different effects. First, in the harmonically unrelated chords and both letters conditions, we see the well-documented but small effect shown by A. S. Reber and others in novel domains. Second, in the harmonically related chords condition, we see the much greater effect of training against a background of previously acquired tacit knowledge.

\section{EXPERIMENT 1B}

An alternative explanation for our results for the harmonically related chords condition in Experiment $1 \mathrm{~A}$ could be that people are already able to classify the sequences of harmonically related chords without any learning at all; so to test this alternative, we conducted an experiment identical to Experiment 1A, except for the absence of the learning phase (see Dienes \& Altmann, 2003; R. Reber \& Perruchet, 2003, for a discussion of measuring learning using an untrained control group of participants).

\section{Method}

Participants. The participants were 90 undergraduate students at Rutgers Newark University who participated in the experiment for course credit or as a course requirement. Participants were randomly assigned to one of three no-training control conditions: harmonically related chord sequences ( $n=30 ; 8$ males, 22 females), harmonically unrelated chord sequences ( $n=30 ; 11$ males, 19 females), or letter sequences ( $n=30 ; 8$ males, 22 females). Participants were excluded if they reported that they played a musical instrument of any kind.

Stimuli. Stimuli were identical to those used in the three relevant conditions of Experiment 1A: harmonically related chords, harmonically unrelated chords, and the related letter set: M, S, W, R, and X. Thirty-six sequences were selected for each condition from among the possible grammatical sequences of each type.

Design. The three conditions were between subjects and were analyzed as independent groups.

Procedure. The procedure was the same as that of Experiment 1A, except that participants were not exposed to the training set. They were informed that they would listen to sequences of chords or letter names and that the sequences belonged to two different groups, each of which was constructed by its own set of rules. Participants were 
asked to guess whether a sequence belonged to Group A or Group B. They were informed that they should trust their "gut feelings" for answering. The test phase encompassed 36 grammatical and 36 ungrammatical sequences.

\section{Results}

A one-way ANOVA performed on the mean classification performance of the three no-training control conditions revealed a nonsignificant effect of experimental condition $\left[F(2,87)=2.58, M S_{\mathrm{e}}=.01, p=.67\right]$, indicating that classification performance was comparable in each no-training control condition. None of the no-training conditions performed significantly differently from chance: related chords $(M=.52, S D=.11)[t(29)=0.82, p=.42, d=$ $.02]$, unrelated chords $(M=.50, S D=.07)[t(29)=-0.19$, $p=.85, d=-.002]$, related letter sequences $(M=.51$, $S D=.05)[t(29)=0.70, p=.49, d=.01]$. Compared with the performance levels seen in Experiment 1A, participants who received no training (Experiment 1B) performed significantly worse: related chords $[t(78)=6.48, p<.001$, $d=.16]$, unrelated chords $[t(78)=5.45, p<.001, d=$ $.10]$, related letters $[t(79)=4.41, p<.001, d=.09]$.

\section{Discussion}

Without training, participants in the harmonically unrelated chords and letters conditions performed at chance levels on the discrimination task and worse than participants in Experiment 1A. We conclude that the superior performance of participants in the harmonically related chord condition in Experiment 1A depended on using domain-specific knowledge to learn a distinction, rather than merely detecting a known distinction between two classes of stimuli.

It could be argued that, because there might be multiple dimensions into which a mixed group of grammatical and ungrammatical sequences could be divided (number of items, presence of a particular item, etc.), chance performance in Experiment 1B was the expected outcome. By that argument, Experiment 1B would not have formed an adequate control for Experiment 1A (see also R. Reber \& Perruchet, 2003).

We thought it was important to include Experiment 1B, however, to show that the grammatical harmonically related chords were not distinct enough to be distinguished from the ungrammatical harmonically related chords without training. A relevant comparison might be to English speakers trying to distinguish sentences that differ in a subtle way, such as containing a particular sequence of dependent clauses. With exposure to a training set, the listener might be able to identify "rule-following" from "rule-breaking" sequences, even though both "rule-following" and "rule-breaking" are grammatical English sentences. They would not, however, be able to do so without both a background knowledge of English syntax and exposure to a training set of "rule-following" sequences.

In order to strengthen the case that participants could not distinguish grammatical from ungrammatical harmonically related chords without the benefit of training on a harmonically related chord set, we included two conditions in Experiment 2 that bear on the issue.

\section{EXPERIMENT 2}

Experiment 2 addressed the second part of our investigation into the effect of prior knowledge on implicit learning: transfer effects. Transfer effects have long been a major focus of theoretical interest because, it has been argued, they show the abstraction of domain-general regularities from learned material (Knowlton \& Squire, 1996; Manza \& Reber, 1997; Mathews, 1990; R. Reber, 1967, 1989).

Because we view implicit learning as ubiquitous but domain-specific, we would not necessarily predict transfer effects between domains, in spite of abstract similarities in the sequences tested. We hypothesize that the salience of structurally identical regularities may differ widely in different domains; for example, the presence of a musically well-formed resolution, C_C, might be salient in a chord sequence domain, whereas the equivalent trigram of letter names could seem entirely unremarkable. This salience would be independent both of position in the sequence and frequency of occurrence in the training set.

We hypothesize that when the patterns of salience in the training material in one domain resemble those in another domain, some transfer will be found, whereas differences in patterns of salience will reduce transfer. We therefore expect better performance in within-domain transfer tasks (letters to letters, or chords to chords) than in cross-domain transfer tasks (chords to letters), which differ more in the salience of particular patterns.

In our study, we examined two kinds of transfer: (1) within-domain transfer from one kind of chord sequence to another (related chords to unrelated chords and unrelated chords to related chords) and from one letter name sequence to another (related letters to unrelated letters and unrelated letters to related letters); and (2) crossdomain transfer from related chords to an analogous letter set, or from related letters to related chords.

We also note the value of two of the conditions in Experiment 2 as controls for Experiment 1B: the unrelated chords to related chords and the related letters to related chords. In both of these conditions, the test set was harmonically related chords, but the training set, although it provided respondents with information about the dimensions along which sequences varied (string length, domain, variability, etc.), was not harmonically related chords. These two conditions, then, test respondents' ability to distinguish grammatical harmonically related chords from ungrammatical harmonically related chords in the absence of domain-relevant training.

\section{Method}

Participants. The participants were 246 undergraduate students at Rutgers Newark University who participated in the experiment for course credit or as a course requirement. None reported that they played a musical instrument of any kind.

Participants were randomly assigned to one of six conditions. Four conditions examined within-stimulus domain transfer effects: harmonically related to harmonically unrelated chords ( $n=40$; 11 males, 29 females); harmonically unrelated to harmonically related chords ( $n=42 ; 15$ males, 27 females); harmonically related letter sequences to harmonically unrelated letter sequences $(n=$ 40; 16 males, 24 females); harmonically unrelated letter sequences 
to harmonically related letter sequences ( $n=41 ; 18$ males, $23 \mathrm{fe}$ males). The remaining two conditions examined cross-stimulus domain transfer effects: harmonically related chords to harmonically related letter sequences ( $n=40 ; 10$ males and 30 females) and harmonically related letter sequences to harmonically related chords ( $n=43 ; 11$ males, 32 females).

Stimuli. Stimuli were identical to those used in relevant conditions of Experiment 1.

Design. The six conditions were completely between subjects.

Procedure. Consistent with previous research on transfer effects in artificial grammar learning, the training phase of Experiment 2 was identical to that of Experiment 1A. During training, the 18 grammatical sequences were repeated four times in a random order, for a total of 72 sequences. The procedures (i.e., length of acquisition phase) used in the present series of experiments were comparable to those of past artificial grammar experiments that have examined transfer effects (e.g., Altmann et al., 1995; Conway \& Christiansen, 2005; Dienes \& Altmann, 1997).

After a 1-min break, participants were informed that the sequences that they had heard were constructed by a set of rules. They were told that they would hear more sequences and that they should decide whether the new sequences were formed by the same set of rules as the sequences they had heard before. Participants in the cross-domain condition were told that the new sequences would now be letter names rather than musical chords (or chords rather than letters), but that they should try to figure out which letter sequences were formed by the same rules that formed the musical sequences they had heard before (or which chord sequences were formed by the same rules that formed the letter sequences they had heard before). As in Experiment 1A, participants pressed "P" for "yes" and "Q" for "no." They were informed that they should trust their "gut feeling" for answering. The test phase encompassed 36 grammatical and 36 ungrammatical sequences.

\section{Results}

Figure 4 shows the mean percentage of correct classifications of each transfer condition. A one-way ANOVA

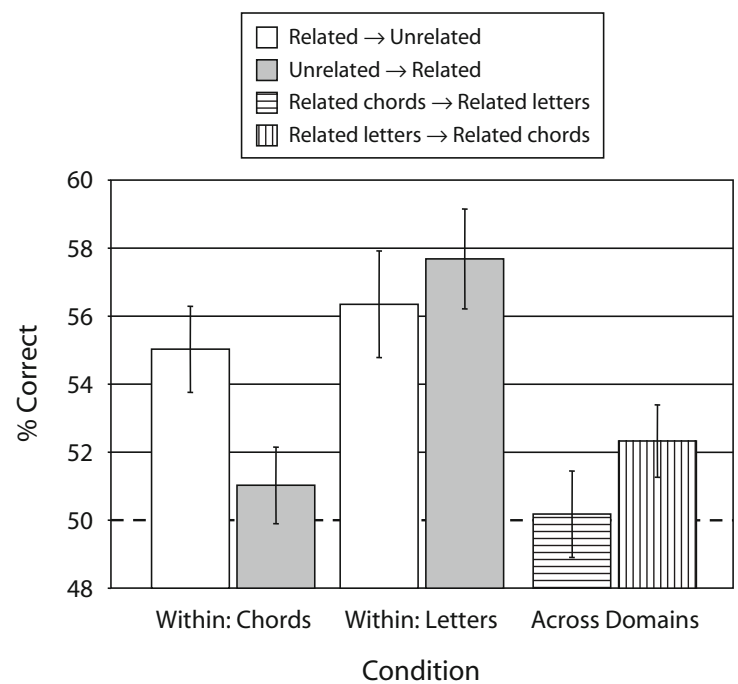

Figure 4. Accuracy of sequence classification in Experiment 2. Solid white bars show transfer from harmonically related to harmonically unrelated within the musical chords and letter name stimulus domains. Solid gray bars show transfer from harmonically unrelated to harmonically related within the musical chords and letter name stimulus domains. Horizontal gray bars and vertical gray bars show transfer between the stimulus domains (related to unrelated). performed on the mean classification performance of the within- and across-domain transfer conditions revealed a significant effect of experimental condition $[F(5,240)=$ $\left.5.43, M S_{\mathrm{e}}=.01, p<.001\right]$. The related- to unrelated-chord condition's discrimination performance was at $55.0 \%$ $(S D=.08)$ correct, significantly better than baseline levels seen in the no-training unrelated (Experiment 1B) condition $[t(68)=2.59, p<.01, d=.04]$. Participants in the unrelated- to related-chord condition performed at 51\% $(S D=.07)$, which was not significantly different from the baseline levels seen in the no-training related (Experiment 1B) condition $[t(70)=-0.27, p=.79, d=-.01]$.

Participants in the related letter sequences to unrelated letter sequences condition performed at $56.4 \%(S D=.10)$, significantly better than the no-training controls (Experiment 1B) $[t(68)=3.13, p<.001, d=.07]$. Performance in the unrelated letter sequences to related letter sequences condition was at $57.7 \%(S D=.09)$, significantly different from performance seen in the no-training control condition $[t(69)=3.93, p<.001, d=.08]$.

Participants in the two cross-domain conditions demonstrated performance indistinguishable from baseline levels seen in Experiment 1B. Performance in the related chords to related letter sequences condition was at $50.2 \%$ ( $S D=$ $.08)[t(68)=0.29, p=.82, d=.004]$. Performance in the related letter sequences to related chords was at $52.3 \%$ $(S D=.07)[t(71)=-0.35, p=.73, d=.01]$.

Awareness data. We assessed subjective awareness for the three conditions that showed significant learning: related chord sequences to unrelated chord sequences, related letter sequences to unrelated letter sequences, and unrelated letter sequences to related letter sequences.

A one-way ANOVA (see Table 3) showed no significant differences in confidence ratings among the three transfer conditions $\left[F(2,118)=1.43, M S_{\mathrm{e}}=.38, p=.24\right]$; neither were participant estimates of classification performance significantly correlated with performance for any condition: related chord sequences to unrelated chord sequences $[r(38)=.20, p=.21]$, related letter sequences to unrelated letter sequences $[r(38)=.05, p=.78]$, and unrelated letter sequences to related letter sequences $[r(39)=.07, p=.65]$.

A one-way ANOVA performed on the difference scores between the certainty ratings for correctly classified and incorrectly classified items for the three conditions (Table 4) found no significant differences among the three transfer conditions $\left[F(2,118)=0.64, M S_{\mathrm{e}}=.05, p=.53\right]$. The only significant difference from zero was present in the unrelated letter sequences to related letter sequences condition $[t(40)=2.41, p<.01, d=.08]$, for related letter sequences to unrelated letter sequences $[t(39)=1.93$, $p=.06, d=.08]$, and for related chord sequences to unrelated chord sequences [t(39) $=0.96, p=.35, d=.03]$. Participants in the letter sequence conditions, therefore, were slightly more confident when they made correct decisions, but their confidence did not predict accuracy.

A one-way ANOVA (see Table 5) performed on the guessing scores (confidence rating of 1) revealed no significant differences among the three transfer conditions $[F(2,103)=$ $\left.0.55, M S_{\mathrm{e}}=.04, p=.58\right]$. Classification performance when 
participants were guessing was not significantly different from chance for each condition: related chord sequences to unrelated chord sequences $[t(34)=1.46, p=.15, d=.04]$, related letter sequences to unrelated letter sequences condition $[t(31)=0.96, p=.35, d=.04]$, and unrelated letter sequences to related letter sequences condition $[t(38)=-0.11$, $p=.91, d=-.004]$. Since participants knew when they were applying knowledge and when they were just guessing, this suggests that they did not acquire implicit knowledge.

A one-way ANOVA comparing the estimates of percent correct among the three transfer conditions revealed no difference $\left[F(2,116)=0.38, M S_{\mathrm{e}}=.43, p=.69\right]$; neither were participant estimates of percent correct correlated with performance: related chord sequences to unrelated chord sequences $[r(38)=.20, p=.21]$, related letter sequences to unrelated letter sequences $[r(38)=.12, p=$ $.48]$, and related letter sequences to unrelated letter sequences $[r(39)=.17, p=.28]$.

\section{Discussion}

The within-domain transfer seen in the related to unrelated chords, related to unrelated letter, and unrelated to related letter sequences are typical of the transfer effects found in the literature: above chance and control levels, but lower than when the same vocabulary is used in training and testing. We did not, however, find transfer between harmonically related chord sequences and analogous letter sequences. Thus, letter discrimination was aided by prior exposure to a different letter set but not by exposure to identically constructed chord sequences. This argues strongly for domain specificity in implicit learning.

Importantly, transfer results were not related to which training conditions might have facilitated better learning (as predicted by results of Experiment 1A). In Experiment $1 \mathrm{~A}$, the harmonically related chord condition might be argued to have been more successfully learned than other conditions. In Experiment 2, however, only one of the two conditions with harmonically related chord training resulted in transfer. In addition, of the three conditions in which letters formed the training set, two showed transfer and the other did not. Transfer performance, then, was not related to which training sets could be better learned, but rather to the interaction between better-learned training sets and similarity between training and test sets.

Two of the conditions in Experiment 2 bore on the topic of Experiment 1B: whether respondents can distinguish grammatical from ungrammatical harmonically related chords without training. The two conditions were the harmonically unrelated chord to harmonically related chord condition and the related letter to related chord condition. Given that neither of these conditions showed abovechance levels of performance on the harmonically related chord test sets, respondents must not have been able to distinguish grammatical from ungrammatical sequences without relevant training.

We found transfer in the harmonically related to harmonically unrelated chord condition, but no transfer in the harmonically unrelated to harmonically related chord sequence condition. One might expect, given that they are both in the musical stimulus domain, that transfer be- tween harmonically related and harmonically unrelated tasks would be as high as, or higher than, the transfer between two letter sets. We suggest that this result speaks of a specificity of learning, revealed in a stimulus domain, that strongly reflects participants' tacit prior domain knowledge. We see transfer in the harmonically related to unrelated chords transfer condition because the harmonically related sequences lead to improved learning. Since the harmonically unrelated chord sequences conflict with prior domain knowledge, training on the harmonically unrelated chord sequences was even harder than training on the arbitrary letter name sequences, with concomitant reduction in transfer effects, even to within-domain harmonically related sequences.

\section{GENERAL DISCUSSION}

\section{Implicit Learning}

Our findings stand in contrast to some classical and neural net theories of implicit learning. According to these accounts, abstract knowledge about positional relationships in harmonically related chord sequences should be accessible in other domains. The connectionist models proposed by Altmann et al. (1995), Cleeremans et al. (1998), and Roussel, Mathews, and Druhan (1990) would predict that if a neural network had learned distributional properties in one domain, it should be possible to transfer this knowledge to another domain, resulting in higher performance, regardless of the effects of domain-specific representational constraints.

The "abstract analogy account" of Brooks and Vokey (1991), Gomez (1997), and Gomez, Gerken, and Schvaneveldt (2000) contends that although implicit learning itself might be supported by abstraction of regularities, transfer would depend on the salience of bigrams and trigrams that could potentially become the basis of participants' explicit knowledge and that beginning position would be a better predictor of salience than frequency of occurrence. Given that all our grammatical sequences are matched in terms of the frequency and position of permissible bigrams and trigrams, the abstract analogy account prediction would seem to be that either we would find transfer effects in none of the conditions, or in all.

Although we do not regard these results as strong evidence for a chunking theory, we are sympathetic to the proposal that the key to understanding transfer may depend on the salience of sequence parts or transitions. For within-domain transfer between two arbitrary tasks- - letter names to letter names, for example - Gomez may be right about positional salience being important. Sequence-initial trigrams may be more salient to participants than other sequence regularities are; therefore, in an artificially abstract task, positional information may be the primary source of constraints available to participants. But positional information is only one of the ways that information can gain salience. Our results suggest that if other, less abstract constraints are available, they are a critical determinant that is not purely dependent on the statistics of pattern elements.

Participants' prior knowledge conferred salience on certain regularities in the harmonically related chord se- 
quences in Experiment 1 that went beyond positional or frequency salience. Thus, the C_C sequences in the harmonically related condition that resolve the cadence have particular salience, because many Western musical themes resolve in a similar way. This salience helped participants discriminate harmonically related grammatical sequences (Experiment 1A), but when the salience was not conferred during the training set, participants could not discriminate harmonically related grammatical sequences (Experiment $1 \mathrm{~B}$ and Experiment 2, harmonically unrelated chords to harmonically related chords condition). Thus we argue that the prior knowledge aided acquisition or discrimination of stimuli only in the same domain, and beyond that, only on a task in the same domain that shared those salience patterns.

Some researchers have argued that a process - implicit learning - that occurs in many domains must be domain general, and some have even proposed a domain-general device akin to a language acquisition device. In contrast, we believe (as do adherents of competitive chunking and neural network models) that there is no need to assume a strict dichotomy between explicit and implicit learning systems. Indices of implicit learning will be found in every domain, not because there is a separate system that represents structure in an abstract way, but because every domain and function relies to a greater or lesser degree on a process of detecting representational regularities in a specific environmental input. The commonality is thus inherent to the computational problem of detecting regularities, not to the underlying machinery that the problem brings into play.

There is evidence from our guessing criterion data that participants may have been aware of their lack of knowledge in cases in which they relied on guessing, but this - in combination with an inability to predict classification success - suggests a particular sort of metaknowledge: awareness of their own lack of certainty on particular trials, rather than an explicit knowledge of classification criteria.

\section{Novel Versus Familiar Domains}

We have argued that the advantage conferred on participants in the harmonically related condition in Experiment $1 \mathrm{~A}$ was a domain-specific advantage caused by their prior familiarity with norms of Western music. In Experiment 2, we showed that the learning advantage in the harmonically related task did not transfer at all to other domains, and transferred only minimally to same-domain tasks that did not share critical regularities.

We hypothesize that this was due to the fact that participants' background knowledge made certain features of the harmonically related sequences salient. By our account, salience is a property not of any particular sequential relationship but of the relationship between the stimulus and preexisting domain knowledge.

In the harmonically related condition, the resolution at the end of the chord sequences was a usual one in Western music; in the harmonically unrelated condition, the repetition structure of the chords was precisely the same, but would not be heard as a resolution. The salient property of the sequence, then, whether or not the ending trigram resolved itself, was dependent not on features of the chord patterns or frequencies, but on participants' prior knowledge. If participants had prior exposure to Western musical norms, they probably recognized the resolution as a salient characteristic of the sequence; if not, that information was not available to them.

For artificial grammar tasks in general, then, performance should be relative to the degree to which participants' experience in the domain highlights for them critical features of the sequences. In the typical artificial grammar tasks in the literature, participants have little relevant information from prior domain exposure. It is our contention that they then rely on weak cues, such as sequence-initial trigram configurations, relative frequency of trigrams, or permissible repetitions, in order to discriminate between grammatical and ungrammatical sequences. In the Critique of Pure Reason, Kant (1781, translated 1998) proposed that our existing conceptual categories must inform the way that that we assimilate experience. In the terms of the present investigation, we find that prior domain knowledge is a lens through which the learning task is perceived, shaping the salience of aspects of novel experience. The effect of prior domain knowledge on learning is therefore crucial; the relationship of new information to conceptual organization and known categories critically affects how we assimilate our experience of the world.

\section{AUTHOR NOTE}

We thank Bridget Bly, Ronny Preciado, and Donovan Rebbechi for their efforts in analysis and interpretation, data collection, and the creation of musical stimuli for this research. We also thank Pierre Perruchet and two anonymous reviewers for their helpful comments. Correspondence concerning this article should be addressed to B. M. Bly (e-mail: benjamin.martin.bly@gmail.com).

\section{REFERENCES}

Aldwell, E., \& Schachter, C. (2003). Harmony and voice leading (3rd ed.). Belmont, CA: Wadsworth.

Altmann, G. T. M., Dienes, Z., \& Goode, A. (1995). Modality independence of implicitly learned grammatical knowledge. Journal of Experimental Psychology: Learning, Memory, \& Cognition, 21, 899-912.

BerRy, D. C., \& DienEs, Z. (1991). The relationship between implicit memory and implicit learning. British Journal of Psychology, 82, 359-373.

Besson, M., \& FAÏTA, F. (1995). An event-related potential (ERP) study of musical expectancy: Comparison of musicians with nonmusicians. Journal of Experimental Psychology: Human Perception \& Performance, 21, 1278-1296.

Besson, M., \& SCHÖN, D. (2003). Comparisons between language and music. In R. Zatorre \& I. Peretz (Eds.), The biological foundations of music (pp. 267-293). Oxford: Oxford University Press.

Bharucha, J. J., \& Stoeckig, K. (1986). Response time and musical expectancy: Priming of chords. Journal of Experimental Psychology: Human Perception \& Performance, 12, 403-410.

Bharucha, J. J., \& Stoeckig, K. (1987). Priming of chords: Spreading activation or overlapping frequency spectra? Perception \& Psychophysics, 41, 519-524.

Brooks, L., \& VOKEY, J. (1991). Abstract analogies and abstracted grammars: Comments on Reber (1989) and Mathews et al. (1989). Journal of Experimental Psychology: General, 120, 316-323.

CARrión, R. E., \& BLY, B. M. (2008). The effects of learning on eventrelated potential correlates of musical expectancy. Psychophysiology, 45, 759-775.

CHAN, C. (1992). Implicit cognitive processes: Theoretical issues and applications in computer systems design. Unpublished doctoral thesis, University of Oxford, Oxford, England. 
Cheesman, J., \& Merikle, P. M. (1984). Priming with and without awareness. Perception \& Psychophysics, 36, 387-395.

Cleeremans, A., Destrebecqz, A., \& Boyer, M. (1998). Implicit learning: News from the front. Trends in Cognitive Sciences, 2, 406-416.

Cleeremans, A., \& McClelland, J. L. (1991). Learning the structure of event sequences. Journal of Experimental Psychology: General, 120, 235-523.

Conway, C. M., \& Christiansen, M. H. (2005). Modality-constrained statistical learning of tactile, visual, and auditory sequences. Journal of Experimental Psychology: Learning, Memory, \& Cognition, 31, 24-39.

Crummer, G. C., Walton, J. C., Wayman, J. W., Hantz, E. C. \& FrISINA, R. D. (1994). Neural processing of musical timbre by musicians, nonmusicians, and musicians possessing absolute pitch. Journal of the Acoustical Society of America, 95, 2720-2727.

Dienes, Z., \& Altmann, G. [T. M.] (1997). Transfer of implicit knowledge across domains: How implicit and how abstract? In D. Berry (Ed.), How implicit is implicit learning? (pp. 107-123). Oxford: Oxford University Press.

Dienes, Z., \& Altmann, G. [T. M.] (2003). Measuring learning using an untrained control group: Comments on R. Reber and P. Perruchet. Quarterly Journal of Experimental Psychology, 56A, 117-123.

Dienes, Z., Altmann, G. T. M., \& GaO, S.-J. (1999). Mapping across domains without feedback: A neural network model of transfer of implicit knowledge. Cognitive Science, 23, 53-82.

Dienes, Z., Altmann, G. T. M., Kwan, L., \& Goode, A. (1995). Unconscious knowledge of artificial grammars is applied strategically. Journal of Experimental Psychology: Learning, Memory, \& Cognition, 21, 1322-1338.

Gomez, R. L. (1997). Transfer and complexity in artificial grammar learning. Cognitive Psychology, 33, 154-204.

Gomez, R. L., Gerken, L., \& Schvaneveldt, R. W. (2000). The basis of transfer in artificial grammar learning. Memory \& Cognition, 28 253-263.

KANT, I. (1998). Critique of pure reason (P. Guyer, Trans.). New York: Cambridge University Press. (Original work published 1781)

Knowlton, B. J., \& SQuire, L. R. (1996). The information acquired during artificial grammar learning. Journal of Experimental Psychology: Learning, Memory, \& Cognition, 22, 169-181.

Koelsch, S., Gunter, T. C., Friederici, A. D., \& Schröger, E. (2000) Brain indices of music processing: "Nonmusicians" are musical. Journal of Cognitive Neuroscience, 12, 520-541.

Koelsch, S., Gunter, T. C., Schröger, E., Tervaniemi, M., \& FrieDERICI, A. D. (2001). Differentiating ERAN and MMN: An ERP study. NeuroReport, 12, 1385-1389.

Koelsch, S., JentschKe, S., SAmmler, D., \& Mietchen, D. (2007). Untangling syntactic and sensory processing: An ERP study of music perception. Psychophysiology, 44, 476-490.

Koelsch, S., Maess, B., Grossmann, T., \& Friederici, A. D. (2003). Electric brain responses reveal gender differences in music processing. NeuroReport, 14, 709-713.

KostKa, S., \& Payne, D. (2000). Harmony, with an introduction to twentieth-century music (4th ed.). New York: McGraw-Hill.
ManZA, L., \& Reber, A. S. (1997). Representing artificial grammars: Transfer across stimulus forms and modalities. In D. C. Berry (Ed.), How implicit is implicit learning? (pp. 73-106). New York: Oxford University Press.

Mathews, R. C. (1990). Abstractness of implicit grammar knowledge: Comments on Perruchet and Pacteau's analysis of synthetic grammar learning. Journal of Experimental Psychology: General, 119, 412-416.

Perruchet, P., \& Pacteau, C. (1990). Synthetic grammar learning: Implicit rule abstraction or explicit fragmentary knowledge? Journal of Experimental Psychology: General, 119, 264-275.

REBER, A. S. (1967). Implicit learning of artificial grammars. Journal of Verbal Learning \& Verbal Behavior, 6, 317-327.

Reber, A. S. (1989). Implicit learning and tacit knowledge. Journal of Experimental Psychology: General, 118, 219-235.

ReBer, A. S. (1993). Implicit learning and tacit knowledge: An essay on the cognitive unconscious. New York: Oxford University Press.

Reber, R., \& Perruchet, P. (2003). The use of control groups in artificial grammar learning. Quarterly Journal of Experimental Psychology, 56A, 97-115.

ReED, J., \& Johnson, P. (1994). Assessing implicit learning with indirect tests: Determining what is learned about sequence structure Journal of Experimental Psychology: Learning, Memory, \& Cognition, 20, 585-594.

Roussel, L. G., Mathews, R. C., \& Druhan, B. B. (1990). Rule induction and interference in the absence of feedback: A classifier system model. In Proceedings of the 12th Annual Conference of the Cognitive Science Society (pp. 733-740). Hillsdale, NJ: Erlbaum.

Servan-Schreiber, E., \& ANderson, J. R. (1990). Learning artificial grammars with competitive chunking. Journal of Experimental Psychology: Learning, Memory, \& Cognition, 16, 592-608.

Steinbeis, N., Koelsch, S., \& Sloboda, J. A. (2006). The role of harmonic expectancy violations in musical emotions: Evidence from subjective, physiological, and neural responses. Journal of Cognitive Neuroscience, 18, 1380-1393.

SwaIN, J. P. (1997). Musical languages. New York: Norton.

Tekman, H. G., \& Bharucha, J. J. (1998). Implicit knowledge versus psychoacoustic similarity in priming of chords. Journal of Experimental Psychology: Human Perception \& Performance, 24, 252-260.

Tervaniemi, M., Ilvonen, T., Karma, K., Alho, K., \& NäÄTÄnen, R. (1997). The musical brain: Brain waves reveal the neurophysiological basis of musicality in human participants. Neuroscience Letters, 226, 1-4.

Tillmann, B., Bharucha, J. J., \& Bigand, E. (2000). Implicit learning of tonality: A self-organizing approach. Psychological Review, 107, 885-913

VOKEY, J. R., \& BRooks, L. R. (1992). Salience of item knowledge in learning artificial grammars. Journal of Experimental Psychology: Learning, Memory, \& Cognition, 18, 328-344.

(Manuscript received May 16, 2006; revision accepted for publication August 25, 2008.) 